\title{
Optimized Research of Energy-saving of the wind system in HVAC based on Improved Ant Colony Algorithm
}

\author{
Professor Shujiang Li,Jingliang Wei,Professor \\ Xiangdong Wang \\ Shenyang University of Technology \\ Institute of information science and technology \\ Shenyang, China \\ e-mail:lisj2005@126.com
}

\begin{abstract}
According to the nonlinear, time-varying variable, large lag and other properties of air handle system, the improved ant colony algorithm is adopted to optimize the air handle system energy consumption. First, mathematical model of wind system of central air conditioning energy consumption is established for improved ant colony algorithm to optimize the model of energy consumption minimization.The improved ant colony algorithm solves the defect of directly deal with continuous optimization problem using ant colony algorithm by discretizing the solution space of continuous function. The experimental results show that, the improved ant colony algorithm can be successfully used in air handle system of HVAC, and has achieved an execellent effect of energy-saving.
\end{abstract}

Keywords- air handle system; energy-saving; improved ant colony algorithm; optimization

\section{INTRODUCTION}

In recent years, the Chinese government pays more attention to energy-saving and emission reduction as well as environmental protection, which has in turn enhanced people's awareness of energy crisis, thus low carbon has become the keyword of national production and rooted in people's daily life. Building energy saving mainly calls for reducing consumption of central air conditioning, and the big shortage of energy in the world as well as the increasing use of air conditioning is taking responsibility for it. ${ }^{1}$

Therefore, reducing the consumption of central air conditioning has become a critical issue in implementing "low carbonization" process. As the consumption of air treatment system accounts for about $35 \%$ of total consumption of air conditioning ${ }^{[1]}$, the research on energy saving of central air conditioning system has attracted more and more attention. $\mathrm{M} \cdot$ Mossolly, $\mathrm{K} \cdot$ Ghali, and $\mathrm{N} \cdot$ Ghaddar, by applying genetic algorithm, proposed a strategy to achieve optimum control of multi-partition air conditioning system-using two improved control strategy to replace the basic control strategy of indoor air quality ${ }^{[2]}$.

Maria Jos é Gacto, Rafael Alcal á and Francisco Herrera, by applying multi-objective evolutionary

Supported by project of Shenyang Municipal Science and Technology Bureau

\author{
Jingliang Wei \\ Shenyang University of Technology \\ Institute of information science and technology \\ Shenyang, China \\ 13940341212@163.com
}

algorithm, optimized the fuzzy logic controller for HVAC. By combining linguistic variables chosen by lateral adjustment rules, they found a way to solve the control problem of HVAC system which is better than the original steady state genetic algorithm for single target ${ }^{[3]}$.

Andrew Kusiak and Guanglin $\mathrm{Xu}$, by using three kinds of improved algorithm of particle swarm, optimized the HVAC energy consumption model for dynamic neural network prediction. According to test result under existing environment of air conditioning system, they found that this strategy could save up as much as $30 \%$ energy compared with traditional control strategy ${ }^{[4]}$.

Shibai Cheng and his team proposed and proved a robust control strategy. They used two independent modules: static pressure reset control and air supply temperature reset control, and applied this control strategy to an office building in Hong Kong, which proved to be more effective compared with traditional air handling unit (AHU) ${ }^{5]}$.

Shuai Liu, Lihua Xie and Wenjian Cai improved the dynamic model of HVAC, and controlled vent and VAV terminal by cooperation, which avoided large overshoot compared with traditional VAV controller ${ }^{[6]}$.

Donghua Zhu, Zhaohui Li, Xiaoxin Tian and Wang Ling, by using total air volume control, put forward the PID control optimization strategy based on ant colony algorithm to solve conventional problems of PID parameter optimization. This strategy, applied in control links of fan's variable frequency and of room temperature, has greatly improved the stability of the system ${ }^{[7]}$.

Xiaolei Kuang, Yuqiang Nie and Angui Li analyzed astringency of the algorithm, from pheromone to volatility coefficient, and conducted controlling structure modification to the system, which proved that it could be effective in energy saving ${ }^{[8]}$. Therefore, the application of control strategy strives to solve the existing problems of high energy consumption in operation and huge useless consumption. Meanwhile, seeing that there is a large room for the improvement of energy saving, intelligent optimization technology can be used to optimize the energy saving for central air conditioning system. Then, by using intelligent optimization algorithms, we can 
optimize the established mathematical model of energy consumption system, output the control parameters of the optimized system, and finally achieve our goal of energy saving.

\section{SETTING UP THE MATHEMATICAL MODEL OF ENERGY CONSUMPTION SYSTEM}

Due to chilled water pump and air supply fan only take low consumption of energy, energy saving can be achieved. The system's objective function and the constraint conditions are as follows:

$$
\min P=P_{\text {pump }}+P_{\text {fan }}
$$

Then polynomial approximation method is applied to define the $P_{\text {pump and }} P_{\text {fan, }, k}$ respectively.

$$
\begin{aligned}
& P_{\text {pump }}=P_{\text {pump }, \text { nom }}\left(d_{0}+d_{1}\left(\frac{m_{w}}{m_{w, \text { nom }}}\right)\right. \\
& \left.-d_{2}\left(\frac{m_{w}}{m_{w, \text { nom }}}\right)^{2}+d_{3}\left(\frac{m_{w}}{m_{w, \text { nom }}}\right)^{3}\right) \\
& P_{\text {fan }}=P_{\text {fan, nom }}\left(e_{0}+e_{1}\left(\frac{m_{a}}{m_{a, \text { nom }}}\right)-\right. \\
& \left.e_{2}\left(\frac{m_{a}}{m_{a, \text { nom }}}\right)^{2}+e_{3}\left(\frac{m_{a}}{m_{a, \text { nom }}}\right)^{3}\right)
\end{aligned}
$$

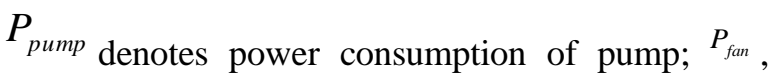
power consumption of AHU fan; $P_{\text {pump,nom }}$, the theoretical power consumption value of pump at full load; $m_{w, n o m}$, the theoretical flow value of chilled water at full load; $P_{\text {fan,nom }}$, the theoretical power consumption value of full load fan; ${ }^{m_{a, n o m}}$, the theoretical flow value of air when fan is under full load; $m_{w}$ and $m_{a}$ stand for the actual flow value of chilled water and the actual flow value of air of fan respectively when air conditioner is at work. $d_{0}$, $d_{1}, d_{2}, d_{3}$ and $e_{0}, e_{1}, e_{2}, e_{3}$ are constant coefficients of pump and fan in operation respectively.

Constraint conditions are as follows

$$
\begin{aligned}
& m_{w, \min } \leq m_{w} \leq m_{w, \max } \\
& m_{a, \min } \leq m_{a} \leq m_{a, \max }(k=1, \ldots, n) \\
& Q_{A H U}=\frac{c_{1} m_{a}^{e}}{1+c_{2}\left(\frac{m_{a}}{m_{w}}\right)^{e}}\left(T_{a i w}-T_{c h w}\right) \\
& Q_{A H U}=c_{w} m_{w}\left(T_{c h w o}-T_{c h w}\right)
\end{aligned}
$$

In this formula, $Q_{A H U}$ denotes cooling load, $T_{\text {aiw }}$ mixed air temperature of $\mathrm{AHU}, T_{c h w}$ the temperature of chilled water supply of $\mathrm{AHU}, T_{\text {chwo }}$ the return temperature of chilled water, ${ }^{c_{1}}$ and $c_{2}$ the parameters to be identified. In this paper, by using penalty function method, we turned the constrained optimization problems to be unconstrained, thus ants can find the optimum solution in the feasible range.

\section{THE ANT COLONY OPTIMIZATION ALGORITHM}

\section{A. The basic ant colony algorithm}

Ant colony algorithm is a new intelligent optimization algorithm proposed by Italy scholars $\mathrm{M} \cdot$ Dorigo, V - Maniezzo and A - Colorni in early 1990 's, which was initially applied to solving the traveling salesman problem (TSP), and made a success. In recent years, the mechanism and principle of this algorithm have attracted researchers' attention. They performed a variety of improvements to the algorithm, which has been successfully applied to solving the scheduling problem, path planning of robot, data mining, machine learning and inference, network routing and various combinatorial optimization problems.

Serap Ulusam Seckiner, Yunus Eroglu, Merve Emrullah, and Turkay Dereli put forward an ant colony optimization algorithm based on pheromone update mechanism which can be applied to continuous function. Every time when the algorithm is iterated, the pheromone will update and determine the number of ants. The test results of 10 basic benchmark functions have showed that this algorithm, compared with the previous one, is more effective in dealing with different types of continuous function ${ }^{[9]}$.

Yuanguo Zhu proposed a new intelligent algorithm combined with mutation ant colony algorithm and simulated annealing algorithm (MACO) to solve the continuous optimization model ${ }^{[10]}$. Garai G., Debbarman S., and Biswas T. put forward a guidance of ant colony algorithm (GACO) applied to optimizing the continuous mathematical function, and the pheromone update mechanism adopted one that would reduce the range of 
search area when iteration number increased. According to the test result, this algorithm showed a better performance compared with other similar ones ${ }^{[11]}$.

\section{B. The improved ant colony algorithm}

Though basic ant colony algorithm can solve discrete space optimization problems well, it still has difficulty in dealing with continuous space optimization problems. This paper adopts the approach of making the continuous space discrete, uses roulette wheel optional method instead of the state transfer probability optional formula of basic ant colony algorithm, and modifies the pheromone update mechanism by using turning pheromone into volatility coefficient algorithm taking from document [8]. The specific steps of the algorithm are as follows

Step 1: Initialization

First solution space is discretized and divided it into a number of subdomains. The initial feasible solution $x_{i}$ $(\mathrm{i}=1,2 \ldots \ldots \mathrm{N})$ will be generated at random, and among all variables of initialization, the pheromone of each ant is represented by ${ }^{\tau_{0}}$, then each ant chooses its initial node (the number of iteration $\mathrm{k}=1$ ).

Step 2: The technique of selecting probability of structural solution path

When the feasible solution domain is not empty, cycle the following formula until the domain turns empty.

$$
p_{i j}^{k}(t)=\left\{\begin{array}{l}
{\left[\tau_{i j}(t)\right]^{\alpha} / \sum_{s \in J}\left[\tau_{i s}(t)\right]^{\alpha}, j \in J} \\
0, \text { otherwise }
\end{array}\right.
$$

In the formula, $\mathrm{J}$ represents feasible solution domain, $\alpha \in(0, \infty)$

When feasible solution domain $\mathrm{J}$ is not empty, cycle formula (1), until $\mathbf{J}$ turns empty, thus the solution construction is completed.

Step 3: Updating pheromone

The update pheromone is as follows: $\tau_{i j}=\left(1-\rho_{n}\right) \tau_{i j}+\Delta \tau_{i j}{ }^{k}$

$\Delta \tau_{i j}{ }^{k}=\left\{\begin{array}{l}Q / f_{k} \\ 0, \text { otherwise }\end{array}\right.$

The No. $\mathrm{k}$ ant passes city $\mathrm{i}$ and city y. Q represents the constant, ${ }^{f_{k}}$ represents fitness of the No. k ant's solution, in formula $\rho_{n}=1-\left(\frac{n}{n+a}\right)^{1 / 2}$ parameter $a>0$

\section{The solution of optimization problem based on improved ant colony algorithm}

It is known that the independent variables in continuous function to be solved are the air supply flow and chilled water flow. This is a two-dimensional continuous problem, several variables are denoted as
$\operatorname{minP}=\Omega=\left\{m_{a}, m_{w}\right\}$, and the range of each component is divided into $\mathrm{N}$ points, leaving $\mathrm{N}$ options. At the beginning we randomly put $\mathrm{M}$ ants in $\mathrm{N}$ cities. When the search starts, each ant moves according to the transfer probability. The layer composed of $\mathrm{N}$ cities in variables to be solved is called layer ${ }^{L_{i}}$, then there are ${ }^{n}$ layers in total. If each ant moves from $L_{1}$ and transfers at every layer until it arrives at $L_{n}$, then the paths ant $\mathrm{K}$ has passed will have a homographic solution $X_{k}$, with that we can work out the corresponding objective function value $\mathrm{F}^{*}$. All the solutions of ants obtained, the pheromone can be updated. .

\section{THE SIMULATION RESULT OF ALGORITHM OPTIMIZATION}

Simulation research for the established central air conditioning system model is built, the data of which is from experimental equipment of central air conditioning system as shown in figure 1 .

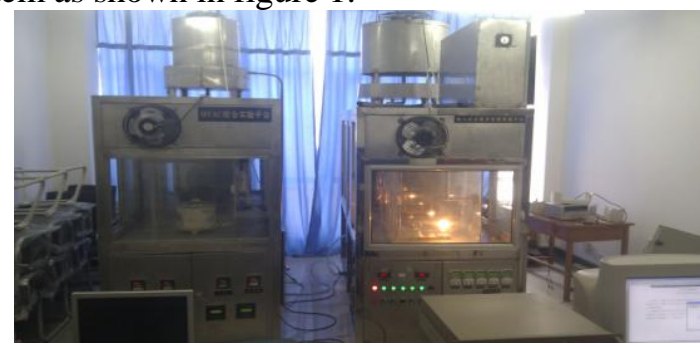

Figure 1. Experimental Platform of central air conditioning system

Central air conditioning system is composed of a chilled water pump, a fan and an air handling unit.

Chilled water pump: rated power $0.73 \mathrm{kw}$;

Air supply fan: rated power $0.28 \mathrm{kw}$

This paper adopts the VAV control system. Suppose cooling load is constant $3.97 \mathrm{kw}$, the temperature of air supply and chilled water supply is set to be constant, $17.8{ }^{\circ} \mathrm{C}$ and $8.13{ }^{\circ} \mathrm{C}$ respectively, and air mass flow rate and chilled water flow are changed to meet the cooling load requirements of the system. $d_{0}, d_{1}, d_{2}, d_{3}$ and $e_{0}, e_{1}, e_{2}, e_{3}$ are $0.00153,0.0052$, $1.1086,-0.116$ respectively. In this condition, we can evaluate the simulation value of ant colony optimization algorithm, and the result is shown in Figure 2.

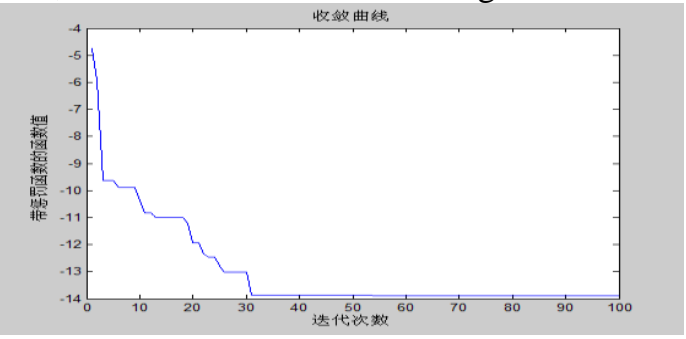

Figure 2. Convergence to improve ants' minimum power consumption 
As we can see in Figure 2, after 53 iterations, curves tend to be steady, and the optimal power consumption has been found out. At this moment, some critical parameters of central air conditioning system can be described as follows: chilled water supply temperature is $7.94{ }^{\circ} \mathrm{C}$, air supply temperature $17.8{ }^{\circ} \mathrm{C}$, chilled water flow $0.64 \mathrm{~L} / \mathrm{h}$, air supply flow $0.257 \mathrm{~kg} / \mathrm{s}$. The optimization searching process of chilled water flow and air mass flow is shown in figure 3 (chilled water pump power is $0.145 \mathrm{kw}$, fan energy consumption is $0.317 \mathrm{kw}$, and the total energy consumption amounts to $0.462 \mathrm{kw}$ ).

Making comparison between the ant colony optimization algorithm proposed in this paper and the basic ant colony algorithm, these two algorithms are used to test the mathematical model of energy consumption of air conditioning system respectively, and thus the result of basic ant colony algorithm after iteration is got, which is shown in Figure 3.

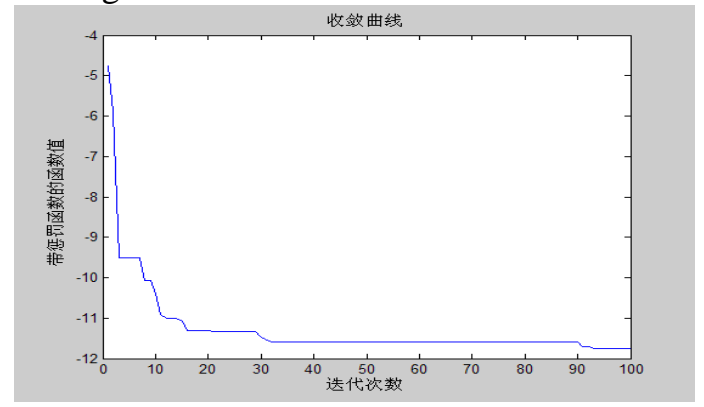

Figure 3. Convergence of basic ant colony algorithm

As seen from Figure 3, the basic ant colony algorithm can be gradually stable until it is iterated about 94 times, and apparently the optimum solution is far from satisfaction, which has been trapped in partial optimum. Therefore, compared with the basic ant colony algorithm, the algorithm proposed in this paper can not only converge on the optimum solution with comparably less iteration, but also effectively avoid falling into partial optimum solution. In conclusion, the improved ant colony algorithm proposed in this paper enjoys two advantagesthe faster speed of convergence and more accurate solution which has been greatly improved.

\section{CONCLUSION}

According to the operation process of central air conditioning system, this paper analyzed the parameters and variables influencing the consumption of air handling unit energy, and confirmed the optimization objective of air treatment system. In addition, to solve the problem that classical optimization algorithm remained uncertain, it proposed to apply ant colony algorithm to energy optimization of air handling system, which brought a satisfactory result - the power consumption of chilled water pump and fan of optimized central air conditioning system have been significantly reduced, and the minimum power of wind system obtained from the experiment also brought us a great success.

\section{REFERENCES}

[1] Quansheng Yuan, Designing and Realizing the Energy-saving System for Central Air-conditioning, $[\mathrm{J}]$, Proceedings of the CSU-EPSA, 2003,15(1):91-93.

[2] M.Mossolly,K.Ghali,N.Ghaddar,Optimal control strategy for a multi-zone air conditioning system using a genetic algorithm[J]. Energy , Volume 34 Issue 1, January 2009, Pages 58-66.

[3] Maria José Gacto,Rafael Alcalá,Francisco Herrera,A multiobjective evolutionary algorithm for an effective tuning of fuzzy logic controllers in heating, ventilating and air conditioning system[J]. Applied Intelligence,Volume 36,Issue 2, pp 330-347,March 2012

[4] Andrew Kusiak , Guanglin Xu, Modeling and optimization of HVAC systems using a dynamic neural network. 8th World Energy System Conference,Volume 42,Issue 1, pp 241-250 June 2012.

[5] Shibai Cheng, Youming Chen, Cary H.K. Chan Lee,Ho Lam Chan,Jianying Qin, Qiang Zhou,Allan Cheung,Kenny Yu,A robust control strategy for VAV AHU system and its application[J].Advanced in intelligent and soft computing,Volume 133,pp635-642,2012

[6] Shuai Liu,Lihua Xie,Wenjian Cai,Cooperative control of VAV air-conditioning systems[C].Control Conference(CCC),page(s)6938-6942,25-27 July 2012

[7] Donghua Zhu,Zhaohui Li,Xiaoxin Tian,Wang Ling,Study of VAV room temperature control based on ant colony algorithm[C], The 2012 Second International Conference on Electric Information and Control Engineering,Volume 1,pages 1584-1587, 2012.

[8] Kuang Xiaolei, Nie Yuqiang, Li Angui, Energy saving performances of central air-conditioning system and its optimal tactics[J], Journal of Harbin Institute of Technology, Volume 41, No.8, 2009.

[9] Serap Ulusam Seckiner, Yunus Eroglu,Merve Emrullah, Turkay Dereli,Ant colony optimization for continuous functions by using novel pheromone updating[J],Applied Mathematic and Computation Volume 219, Issue 9,pages 4163-4175,1 January 2013

[10] Yuanguo Zhu, An intelligent algorithm: MACO for continuous optimization models[J]. Journal of Intelligent and Fuzzy Systems ,Volume 24, pages 31-36,Number 1 / 2013

[11] Garai, G.,Debbarman, S. , Biswas, T. An efficient Ant Colony Optimization algorithm for function optimization[C]. Evolutionary Computation (CEC), 2013 IEEE Congress,pages 2345-2351,20-23 June 2013 